\title{
Application of MDT mode in the diagnosis and treatment of Coronavirus Disease 2019 (COVID-19) Pneumonia
}

\author{
Xujun $\mathrm{FENG}^{1 *}$, Ying $\mathrm{LI}^{2 *}$, Gongyou XIONG ${ }^{3 *}$ (D), Guoqiang TONG ${ }^{1}$, Xiaoming XIONG ${ }^{4}$, Tao HONG ${ }^{2}$, Yun $\mathrm{LUO}^{5}$
}

\begin{abstract}
To explore the effect of Multi-Disciplinary Team (MDT) mode in the diagnosis and treatment of Coronavirus Disease 2019 (COVID-19) Pneumonia. A total of 65 patients with suspected COVID-19 pneumonia were included. On February 8, 2020, our hospital officially became a designated hospital for the treatment of COVID-19, and the MDT mode was implemented throughout the diagnosis and treatment for newly admitted patients with suspected COVID-19. The patients were divided into control group and observation group according to whether received MDT mode. Our results showed that the diagnosis time in the observation group was significantly shortened than that in the control group (2.51 days vs. 3.47 days) $(p<0.05)$. The average daily hospitalization costs in the observation group was significantly decreased in comparison with the control group (¥766.1 vs. $¥ 1190.4)$ ( $\mathrm{p}<0.001)$. The average daily cost of protective materials in the observation group was significantly reduced in comparison with the control group ( $¥ 4226.90$ vs. $¥ 5308.20)(\mathrm{p}<0.001)$. Compared with the control group, the subjective symptoms of patients in the observation group were significantly improved $(\mathrm{p}<0.001)$. In conclusion, the MDT mode shortens the diagnosis time of, reduces the hospitalization costs, and improves the subjective symptoms of COVID- 19.
\end{abstract}

Keywords: COVID-19; MDT mode; subjective symptom; diagnosis time; hospitalization costs.

Practical Application: Multi-Disciplinary Team (MDT) mode shortens the diagnosis time of, reduces the hospitalization costs, and improves the subjective symptoms of COVID-19. MDT mode can be widely applied during COVID-19.

\section{Introduction}

Since December 2019, a number of patients with Coronavirus Disease 2019 (COVID-19) Pneumonia have been found in Wuhan, Hubei Province, China. With the progress and spread of the epidemic, COVID-19 cases have been also found in other areas of China. At present, COVID-19 has been included as an acute respiratory infectious disease into the Class B infectious disease and is managed in accordance with the Class A infectious disease (Huang et al., 2020; China, 2020a). The diagnosis of COVID-19 mainly depends on the positive detection of the viral nucleic acid (Duan \& Qin, 2020; China, 2020b). President Xi of China emphasized that "doing a good job in the prevention and control of the epidemic is directly related to the public safety and health, the overall economic and social stability, and reform and opening". Jiujiang City, the north gate of Jiangxi Province, is located in the junction center of Jiangxi, Hubei and Anhui provinces, and is facing unprecedented anti-epidemic pressure. As a general hospital, it has also become the focus of our hospital's work that how to improve the efficiency of anti-epidemic and to reduce the anti-epidemic cost on the basis of ensuring zero errors in the diagnosis and treatment process.
Multi-Disciplinary Team (MDT) mode is a leading diagnosis and treatment mode that is widely respected in modern medical field. MDT mode has been first proposed in 1997. At the beginning of the 21st century, Anderson first applied electronic medical records and information-based medicine to push MDT into a new era. Specialist can grasp the medical information of patients using the network and information-based medical platform anytime and anywhere. COVID-19 has the features of strong infectivity, noticeable epidemiological characteristics and family clustering infection. The asymptomatic infection may be the major way of infection for people around world. The most common clinical manifestation of COVID-19 is fever, but food handlers' awareness of food risks needs to be notified alongside any updates regarding the COVID-19. So, the diagnosis and treatment should be joined with the epidemiological data and CT imaging findings to prevent misdiagnosis (Gao et al., 2021; Dang \& Dam, 2021) MDT breaks the barriers between disciplines, effectively promotes the construction of disciplines, realizes the joint improvement of doctors, departments and hospitals (Liu et al., 2007; Nikolovski et al., 2017; Slavova-Azmanova et al.,

\footnotetext{
Received 28 Jun, 2021

Accepted 12 Jul, 2021

${ }^{1}$ Department of Respiratory Medicine, Jiujiang First People's Hospital, Jiujiang, Jiangxi, China

${ }^{2}$ Medical Department, Jiujiang First People's Hospital, Jiujiang, Jiangxi, China

${ }^{3}$ President, Jiujiang First People's Hospital, Jiujiang, Jiangxi, China

${ }^{4}$ Infectious Disease Department, Jiujiang First People's Hospital, Jiujiang, Jiangxi, China

${ }^{5}$ Vice President, Jiujiang First People's Hospital, Jiujiang, Jiangxi, China

\#Co-First Authors

*Corresponding author: xionggy404@163.com
} 
2015; Nazim, 2018). Therefore, we aimed to explore the effect of MDT mode in the diagnosis and treatment of COVID-19.

\section{Subjects and methods}

\subsection{Subjects}

This study was approved by the Ethics Committee of the Jiujiang NO.1 People's Hospital. All patients had signed informed consent. A total of 65 patients with suspected COVID-19 who admitted to the Department of Infectious Diseases in the Jiujiang NO.1 People's Hospital from January 17, 2020 to March 4, 2020 were included in the retrospective study. Diagnostic criteria: the diagnosis and treatment plan of COVID-19 pneumonia (trial version fifth and sixth), which was released by the National Health Committee (China, 2020a). On February 8, 2020, our hospital officially became a designated hospital for the treatment of COVID-19, and MDT mode was implemented throughout the diagnosis and treatment for newly admitted patients with suspected COVID-19. The patients were divided into control group and observation group according to whether received MDT mode.

\subsection{MDT intervention}

The expert group on diagnosis and treatment of COVID-19 was set up and approved by the Jiujiang Health Protection Committee. The composition of MDT members was determined by the functions of the participants in the team. The team members include 2 physicians in the department of infectious diseases, 2 physicians in the department of respiratory tract, 2 physicians in the ICU, 2 physicians in the medical imaging department, 2 technicians in the department of clinical laboratory and 1 nutritionist. The head of the group is the business director. At the same time, a preparatory group was set up with 1 physician and 1 surgeon as members.

The training of members includes not only the study of professional knowledge, but also the ability of working together. The leader develops the training courses based on the characteristics of COVID-19 after fully soliciting the opinions of all members. The course includes the latest version of diagnosis and treatment plan of COVID-19, imaging findings of community-acquired pneumonia, pharmacological actions, adverse reactions of antiviral drugs, pharmacological actions and adverse reactions of antimicrobial agents. At the same time, the clinical data of 10 patients with severe community-acquired pneumonia were retrospectively analyzed for simulation training. The members evaluated the effect of MDT mode on community-acquired pneumonia, and discussed the experience in the process of diagnosis and treatment, the allocation of responsibilities, the form of discussion, and the emergency treatment process. The flow chart of MDT mode was shown in Figure 1.

\subsection{Intervention in the control group}

In accordance with the normal procedures and programs, the routine consultation mode was performed. It was divided into two steps, in-hospital consultation and out-of-hospital consultation. It was proposed by the management physician of the department of infection disease and agreed by the chief physician. It was proposed to the medical department for inhospital consultation of each sub-specialist as needed. According to the conventional consultation system, each specialist wrote the consultation opinions, and the management physician reported the consultation opinions to the superior physician, and the chief physician made the preliminary diagnosis. After reporting to the medical department, the department of infection disease applied for out-of-hospital consultation by the municipal expert group. According to the consultation opinions, the suspected cases were removed and transferred to the fever wards. The confirmed patients were transferred to other designated hospitals for treatment.

\subsection{Collection of general information}

The general information of patients were collected, including name, gender, age and occupation. After consulting the literature and discussing, the group members collected them into a general information table (Zhao \& Wu, 2018).

\subsection{Evaluating indicators}

Objective indicators: admission time, discharge time, diagnosis time, total hospitalization costs, daily use of protective materials (protective clothing, N95 mask, goggles, disinfectant, etc.). The diagnosis time, average daily hospitalization costs (¥/day) and material use costs (¥/day) were calculated.

Subjective index: subjective symptom score. Because patients' self-evaluation of the treatment effect is a subjective feeling, it needs a relatively objective scoring method. Referring to the international pain number rating system (NRS), we also set the number from 0 to 10 , which means from invalid (0 points) to very effective (10 points). One number was selected by the patient to show the therapeutic effect. The subjective symptom grading criteria were: 7-10 points as effective; $4-6$ points as improved; $1-3$ points as stable; 0 points as invalid.

\subsection{Statistical analysis}

The statistical software SPSS 21.0 was used for data analysis. The measurement data of normal distribution were expressed as mean \pm standard deviation (SD), and two independent samples $t$-test was used for comparison. The measurement data of nonnormal distribution were expressed as median (p25-p75), and non-parametric Mann Whitney U test was used for comparison. The counting data were expressed as percentage, and chi square test was used for comparison between groups. The difference was statistically significant when $p<0.05$.

\section{Results}

\subsection{Comparison of the general information between the two groups}

There was no significant difference in gender, age and occupation between MDT group and control group $(p>0.05)$ (Table 1). 


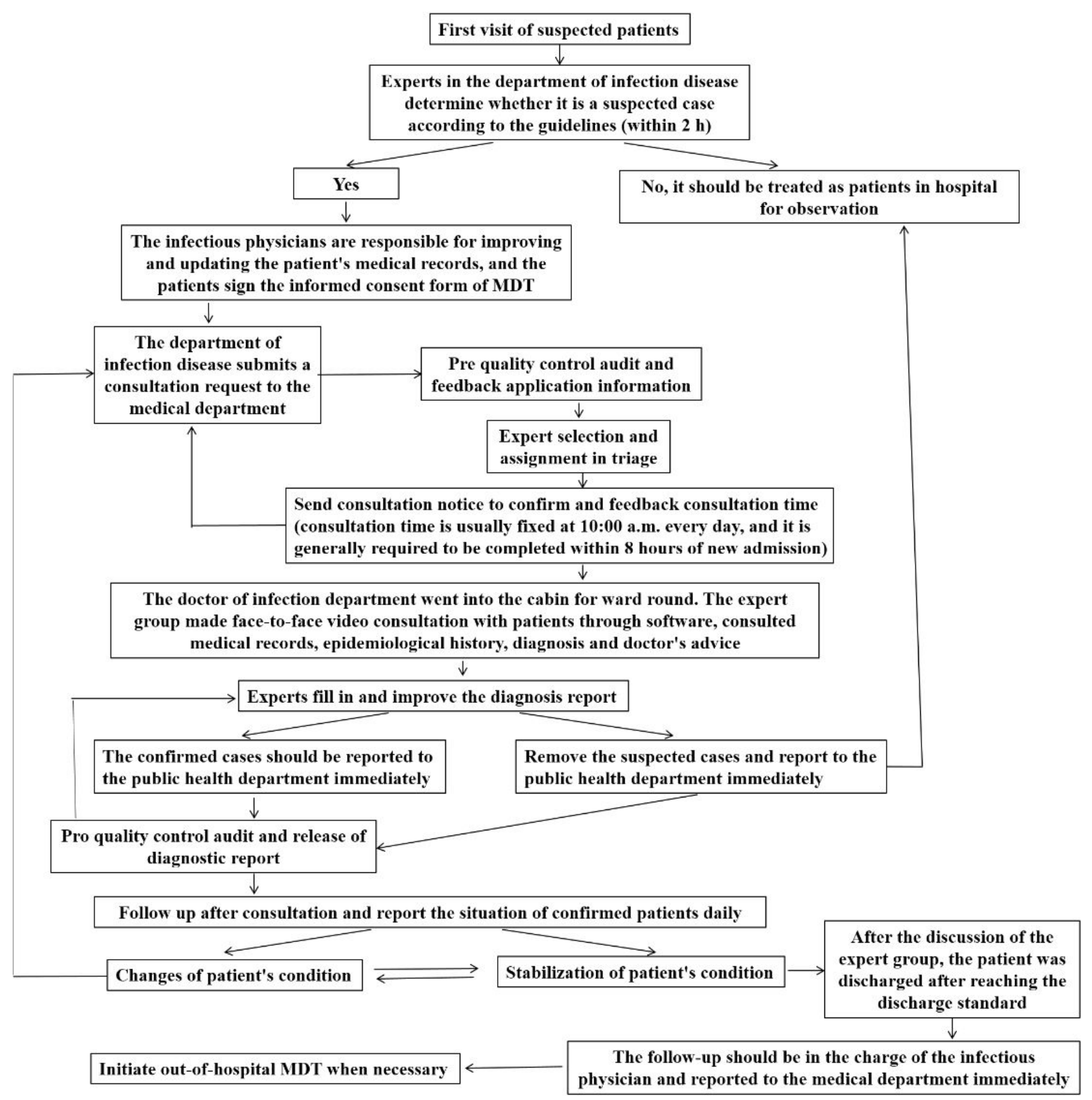

Figure 1. The flow chart of MDT mode.

Table 1. Comparison of general information between the two groups.

\begin{tabular}{|c|c|c|c|c|c|}
\hline & \multirow{2}{*}{ Items } & MDT group & Control group & \multirow{2}{*}{$\chi^{2}$ values } & \multirow{2}{*}{$p$ values } \\
\hline & & $(\mathrm{n}=33)$ & $(\mathrm{n}=32)$ & & \\
\hline \multirow[t]{2}{*}{ Gender } & Male & 14 & 19 & 1.868 & 0.172 \\
\hline & Female & 19 & 13 & & \\
\hline \multirow[t]{3}{*}{ Age (years old) } & $<40$ & 12 & 14 & 1.182 & 0.554 \\
\hline & $40 \sim 60$ & 11 & 12 & & \\
\hline & $>60$ & 10 & 6 & & \\
\hline \multirow[t]{4}{*}{ Occupation } & Worker & 3 & 4 & 4.610 & 0.201 \\
\hline & Farmer & 10 & 5 & & \\
\hline & Administrative institutions & 10 & 6 & & \\
\hline & other & 10 & 17 & & \\
\hline
\end{tabular}




\subsection{Comparison of the confirmed time between the two groups}

The diagnosis time in the observation group was significantly less than that in the control group (2.51 days vs. 3.47 days) $(t=2.35 ; p<0.05)($ Table 2$)$.

\subsection{Comparison of the costs between the two groups}

The average daily hospitalization costs in the observation group was significantly decreased in comparison with the control group ( $¥ 766.1$ vs. ¥1190.4) $(p<0.001)$ (Table 3). The average daily cost of protective materials in the observation group was significantly reduced in comparison with the control group $(¥ 4226.90$ vs. $¥ 5308.20)(p<0.001)$ (Table 3).

\subsection{Comparison of the subjective symptom scores between the two groups}

The efficacy score in the MDT group was 8 (7-8), and that in the control group was 6 (4-7). Compared with the control group, the subjective symptoms of patients in the observation group were significantly improved $(p<0.001)$ (Table 4$)$.

\section{Discussion}

COVID-19 is a public health emergency. How to handle it efficiently has become the focus of current work in our hospital. When our hospital was not a designated hospital, all suspected cases were treated according to the traditional process of consultation of in-hospital and out-of-hospital. In our daily clinical work, we have found that the consultation process is cumbersome and consumes a lot of human resources. The consultation experts only

Table 2. Comparison of confirmed time between the two groups.

\begin{tabular}{lcc}
\hline \multicolumn{1}{c}{ Items } & Number & Confirmed time \\
\hline MDT group & 33 & $2.51 \pm 1.58$ \\
Control group & 32 & $3.47 \pm 1.68$ \\
$t$ values & & 2.35 \\
$p$ values & & 0.022 \\
\hline
\end{tabular}

Table 3. Comparison of costs between the two groups.

\begin{tabular}{lccc}
\hline \multicolumn{1}{c}{ Items } & Number & $\begin{array}{c}\text { Daily } \\
\text { hospitalization } \\
\text { costs }\end{array}$ & $\begin{array}{c}\text { Daily cost of protective } \\
\text { materials }\end{array}$ \\
\hline MDT group & 33 & $766.1 \pm 293.4$ & $4226.90(3932.0-4521.8)$ \\
Control group & 32 & $1190.4 \pm 515.2$ & $5308.20(4915.00-5358.50)$ \\
$t$ values & & 4.096 & - \\
$p$ values & & $<0.001$ & 0.000 \\
\hline
\end{tabular}

Table 4. Comparison of subjective symptom scores between the two groups.

\begin{tabular}{lcc}
\hline \multicolumn{1}{c}{ Items } & Number & $\begin{array}{c}\text { Subjective symptom } \\
\text { scores }\end{array}$ \\
\hline MDT group & 33 & $766.1 \pm 293.4$ \\
Control group & 32 & $1190.4 \pm 515.2$ \\
$p$ values & - & 0.001 \\
\hline
\end{tabular}

have provided subspecialty guidance on the patient's condition, and have not participated in the diagnosis, treatment and later follow-up of the patient throughout the process. The traditional consultation process leads to repeated laboratory examination and imaging examination, which prolongs the diagnosis time. At the same time, it consumes a lot of medical resources and costs high hospitalization expenses.

MDT is a new type of medical mode. MDT mode is guided by the concept of evidence-based medicine, based on a large amount of literature and guidelines of diagnosis and treatment (Collins \& Skilton, 2004; Cao et al., 2007; Liu et al., 2007; Tan et al., 2018). MDT has long been widely used in the departments of ICU, oncology, and surgery (Ye \& Wang, 2011; Hua \& Hong, 2015; Yang et al., 2019). MDT has the characteristics of content standardization, procedure standardization, organization diversification, mode diversification and form informatization, which leads to high efficiency of disease diagnosis and treatment and low medical cost.

Our hospital has developed the MDT mode for many years with standardized MDT system and process, and we have accumulated rich clinical experience at the same time (Sun et al., 2019). In combination with this epidemic, we have formulated a MDT mode that meets COVID-19 in a short period according to the basic conditions of the hospital's hardware facilities and technical capabilities. Through the implementation, feedback, correction, re-implementation, re-feedback, re-correction and other measures, MDT mode has been continuously improved.

Through the application of MDT mode in the diagnosis and treatment of COVID-19, the diagnosis time has been shortened and the average daily hospitalization cost has been reduced. Our results showed that the diagnosis time in the MDT group was 2.51 days, and that in the control group was 3.47 days. Compared with the control group, the diagnosis time in the MDT group was shortened by nearly one day on average, which significantly improved the speed of diagnosis and treatment. At the same time, compared with the control group, the application of the MDT mode reduced the average daily hospitalization costs by about $¥ 400$. When using the traditional consultation process, the average daily costs of epidemic prevention materials were about $¥ 5308.20$. After adopting the MDT mode, the average daily costs of epidemic prevention materials were decreased to $¥ 4226.90$. Furthermore, the subjective symptom score of patients with MDT intervention was 8 points, and that of patients without MDT intervention was 6 points, showing that MDT intervention was significantly effective.

Through the standardized process of MDT, the fixed time and place of MDT are clarified, senior specialists are strictly selected, the participants are relatively fixed, and the team cooperates in diagnosis and treatment. The opinions need to be considered comprehensively, and the problems are found in many aspects as soon as possible to intervene, and the diagnosis and treatment results shall be followed up throughout the process, and the plan shall be revised in a timely manner. Patients have reduced the number of repeated examinations and shortened the time for laboratory examinations and imaging examinations. Targeted auxiliary inspections and reasonable planning of the number of warehousing and the list of warehousing personnel can reduce 
the use of unnecessary protective materials, save the medical resources medical costs. After diagnosis, the expert group jointly formulates individualized treatment plans for different patients, and treats the patients' accompanying diseases and chronic diseases as soon as possible. At the same time, MDT is dominated by experts, but all participating members can put forward their own opinions and unique insights. In contrast to the traditional consultation mode, the attending doctor applies for specialist consultation after encountering problems according to the patient's condition in the process of diagnosis and treatment. The consultation staff was not fixed, and did not receive systematic and standardized training of COVID-19. Only the consultation recommendations that accords with this specialty were put forward, and they are not specifically involved in the diagnosis and treatment decisions of patients. Meanwhile, the cured patients with COVID-19 can continue to obtain the opinions of many experts through MDT clinic after discharge, which is not possessed by the traditional consultation mode.

It should be noted that this study is a single-center retrospective study with a small sample size. It should take into account the characteristics of the local epidemic, medical technical conditions and hardware levels, formulate a practical and realistic diagnosis and treatment model (Shu et al., 2020).

By applying the MDT mode to the diagnosis and treatment of COVID-19, it shortens the diagnosis time and reduced the hospitalization costs. At the same time, through the early removal of suspected cases, the human and material investment required by the local Center for Disease Control (CDC), public security and other departments for prevention and control management have been reduced, the use of social resources has been reduced, and the overall efficiency of the prevention and treatment of COVID-19 has been improved. We describe the exploration of the diagnosis and treatment of COVID-19 in our hospital, so as to provide the experience of major public health emergencies that may occur in the future.

\section{Conflict of interest}

The author declare that they have no competing interest.

\section{Funding}

This study was supported by Science and Technology Plan of Health Commission of Jiangxi Province (No. 20197101).

\section{References}

Cao, L., Wang, X., \& Li, L. (2007). Discussion on consultation process of multidisciplinary collaborative diagnosis and treatment model. Chinese Journal of Basic and Clinical Medicine of General Surgery, (3),343-345.

China, National Health Committee - NHC. (2020a). Announcement of the State Health Commission of the people's Republic of China. Retrieved from h https://xueshu.baidu.com/usercenter/paper/sho w?paperid=e4b9ab611a3b4c23f79b46cdcfdea244\&site=xueshu_se

China, National Health Committee - NHC. (2020b). New Coronavirus pneumonia diagnosis and treatment plan. Retrieved from www.nhc. gov.cn/yzygj/s7653p/202002/8334a8326dd94d329df351d7da8aefc2/ files/b218cfeb1bc54639af227f922bf6b817.pdf
Collins, J., \& Skilton, K. (2004). Low visi on services in South Devon: a multi-agency, multi-disciplinary approach. Ophthalmic \& Physiological Optics, 24(4), 355-359. http://dx.doi.org/10.1111/j.14751313.2004.00225.x. PMid:15228515.

Dang, H. D., \& Dam, A. H. T. (2021). Determinants of hygienic handling of food by consumers in the COVID-19 pandemic context: a crosssectional study in Vietnam. Food Science and Technology, http:// dx.doi.org/10.1590/fst.30221. [Ahead of Print].

Duan, Y. N., \& Qin, J. (2020). Pre- and posttreatment chest CT findings: 2019 novel coronavirus (2019-nCoV) pneumonia. Radiology, 295(1), 21. http://dx.doi.org/10.1148/radiol.2020200323. PMid:32049602.

Gao, T., Xu, Y., He, X., Xu, X., Wang, L., Jiang, Y., Wu, C., \& Zhang, W. (2021). Epidemiological and clinical characteristics of 40 cases of COVID-19 outside Hubei Province, China. Food Science and Technology. [Ahead of Print].

Hua, C., \& Hong, H. (2015). Current situation and prospect of multidisciplinary cancer consultation. Medical Review, 21(3), 431-434.

Huang, C., Wang, Y., Li, X., Ren, L., Zhao, J., Hu, Y., Zhang, L., Fan, G., Xu, J., Gu, X., Cheng, Z., Yu, T., Xia, J., Wei, Y., Wu, W., Xie, X., Yin, W., Li, H., Liu, M., Xiao, Y., Gao, H., Guo, L., Xie, J., Wang, G., Jiang, R., Gao, Z., Jin, Q., Wang, J., \& Cao, B. (2020). Clinical features of patients infected with 2019 novel coronavirus in Wuhan, China. Lancet, 395(10223), 497-506. http://dx.doi.org/10.1016/ S0140-6736(20)30183-5. PMid:31986264.

Liu, Z., Wang, X., \& Li, L. (2007). Current situation of introducing multidisciplinary collaborative management model in colorectal cancer. Chinese Journal of Basic and Clinical General Surgery, 14(1), 114.

Nazim, S. M., Fawzy, M., Bach, C., \& Ather, M. H. (2018). Multidisciplinary and shared decision-making approach in the management of organ-confined prostate cancer. Arab Journal of Urology, 16(4), 367-377. http://dx.doi.org/10.1016/j.aju.2018.06.008.

Nikolovski, Z., Watters, D. A., Stupart, D., \& Guest, G. D. (2017). Colorectal multidisciplinary meetings: how do they affect the timeliness of treatment. ANZ Journal of Surgery, 87(10), E112-E115. PMid:25913227.

Shu, C., Luo, M., Fang, K., Tian, C., Zhang, Y., Xue, Y., Zhao, J., Li, Y., Wu, L., Liu, X., Qiu, X., \& Dong, N. (2020). Recommendations for diagnosis and treatment of aortic emergency in the period of New Coronavirus pneumonia prevention and control. China Circulation Journal, 35(4), 331-337.

Slavova-Azmanova, N. S., Johnson, C. E., Platell, C., Bydder, S., \& Saunders, C. M. (2015). Peer review of cancer multidisciplinary teams: is it acceptable in Australia. The Medical Journal of Australia, 202(3), 144-147. http://dx.doi.org/10.5694/mja14.00768. PMid:25669477.

Sun J., Zhang, C., Luo, Y., Hong, T., \& Xiao, T. (2019). Research on improving the level of AMS by MDT. Contemporary Medicine. 25(31), 121-123.

Tan, S., Li, L., Qiu, M., Zhang, L., Qu, Y., \& Weihong, L. V. (2018). Effect of multidisciplinary collaboration combined with cluster management on the prevention of multi drug resistant bacteria infection in ICU. Chinese Journal of Infection Control, 17(02), 156-159.

Yang, W., Fei, D., Kang, K., Bo, H., Guo, J., Guo, Q., \& Zhao, M. (2019). Application of MDT diagnosis and treatment mode in ICU Teaching Mode Reform. China Continuing Medical Education, 11(22), 1-3.

Ye, Y., \& Wang, S. (2011). Organization and standard implementation of diagnosis and treatment mode of multidisciplinary expert group. Chinese Journal of Practical Surgery., 31(01), 22-24.

Zhao, Y., \& Wu, J. (2018). Study on the application of multidisciplinary collaborative intervention in the targeted treatment of patients with advanced renal cancer at home. Chung-Hua Hu Li Tsa Chih, 53(04), 394-398. 